\title{
$\checkmark$ Research Square \\ Hypotension during hip fracture surgery and postoperative morbidity: a retrospective analysis
}

\section{Rachael Cusack ( $\nabla$ rqcusack@gmail.com )}

Mater Misericordiae University Hospital https://orcid.org/0000-0003-4456-7220

\section{Gabriel Beecham}

Mater Misericordiae University Hospital

\section{Grace Crilly}

University College Dublin

\section{Sebastian Vencken}

University College Dublin

\section{Donal Buggy}

Mater Misericordiae University Hospital

\section{Research article}

Keywords: Hip Fracture, surgery; Hip Fracture, outcome; Anaesthesia, spinal, Anaesthesia, general.

Posted Date: August 14th, 2019

DOI: https://doi.org/10.21203/rs.2.12877/v1

License: (c) (i) This work is licensed under a Creative Commons Attribution 4.0 International License. Read Full License 


\section{Abstract}

Background: Hip fracture is a growing healthcare challenge, with 6-8\% 30-day mortality and $20-30 \%$ of patients incurring major morbidity. Intraoperative hypotension during hip fracture surgery is associated with increased 30-day mortality regardless of anaesthetic technique. Although a recent trial demonstrated reduced postoperative complications by maintaining intraoperative arterial blood pressure close to baseline, there are no data correlating intraoperative hypotension during hip fracture surgery with postoperative morbidity. Objective: We evaluated the hypothesis that duration and severity of intraoperative hypotension during hip fracture surgery is associated with increased postoperative morbidity. Methods: A retrospective analysis was carried out on $n=52$ patients undergoing hip fracture surgery between January and June 2017. Electronic measurements of patients' intraoperative systolic arterial pressure (SAP) and mean arterial pressure (MAP) during anaesthesia were reviewed. We calculated the total duration of time where SAP or MAP were below pre-defined thresholds for hypotension (MAP $<75 \mathrm{mmHg}$, MAP $<55 \mathrm{mmHg}$, SAP $\leq 80 \%$ admission baseline, or SAP $\leq 80 \%$ preinduction baseline). Univariate and bivariate descriptive statistics were generated for all relevant variables. With multivariable regression models containing known predictors, cumulative duration of hypotension was correlated with postoperative comorbidities as quantified by the Clavien-Dindo and Comprehensive Complication Indices. Results: Mean age ( \pm SD) was $78 \pm 13$ years, $75 \%$ were female, $87 \%$ were ASA II or III and 60\% underwent spinal anaesthesia. Mean Comprehensive Complication Index was $20.4 \pm$ 19.2. Lowest absolute SAP and MAP values were $82 \pm 18 \mathrm{mmHg}$ and $55 \pm 12 \mathrm{mmHg}$ respectively. Cumulative time of SAP $<80 \%$ pre-induction value adjusted to gender, age and the Charlson Comorbidity Index was associated with progression to a higher Clavien-Dindo classification (odds ratio: 1.020 per additional minute; $95 \% \mathrm{Cl}: 1.008-1.035 ; \mathrm{P}=0.003)$. Conclusions: In this exploratory retrospective analysis, the cumulative time of hypotension during hip fracture surgery correlated with extensive postoperative morbidity when adjusting to other known predictors. Intraoperative cumulative time of hypotension may be a good candidate for larger prediction studies as a predictor of postoperative complications. A randomised controlled trial evaluating active control of intraoperative hypotension on postoperative morbidity in hip fracture patients seems warranted.

\section{Background}

Hip fracture is a common injury, associated with increased risk of death and major morbidity. 3,629 patients were treated for hip fracture in Ireland in 2016. ${ }^{1}$ The problem is recognised internationally, with 65,000 cases in the United Kingdom in 2016 (costing its National Health Service over $£ 1$ billion) and 321,708 cases in the United States in 2014. 2,3 There were 2.7 million fragility fractures in 2017 across France, Germany, Italy, Spain, the United Kingdom and Sweden, costing $€ 37$ billion and with this cost estimated to increase to $€ 47$ billion by $2030 .{ }^{4}$ The average age of hip fracture patients is 79 years for men and 81 years for women, with $75 \%$ of patients being female. More than half of patients presenting with hip fracture are class III on the American Society of Anesthesiologists (ASA) physical status classification; $7 \%$ are ASA IV, which is associated with a 30 -day mortality of $20 \%$. ${ }^{1,5}$ 
Internationally, the 30-day mortality following hip fracture surgery is $6-8 \%$, a figure which is independent of anaesthetic technique. ${ }^{6}$ Hip fracture is one of the commonest reasons for admission of elderly patients to hospital, and compared with elective total hip replacement it is associated with significant postoperative morbidity including myocardial infarction, cerebrovascular injury, heart failure, renal failure and sepsis. ${ }^{7}$ Patients suffering hip fractures have been demonstrated to have a high burden of preoperative comorbidity, increasing the risk of major postoperative complications. ${ }^{8}$

Early surgical repair (within 24 hours) has been shown to reduce mortality and morbidity in a populationbased cohort study. ${ }^{9}$ While regional anaesthesia has been hypothesised to be beneficial, recent metaanalyses of observational studies have not demonstrated any benefit of regional versus general anaesthesia on 30-day mortality, although the former may confer a small reduction in length of stay. ${ }^{10}$

Hypotension occurs commonly during hip fracture repair, especially in the case of older patients with greater numbers of comorbidities. ${ }^{11}$ A prospective observational study showed that $89 \%$ of patients experienced intraoperative hypotension during hip fracture surgery. ${ }^{5}$ One recent randomised controlled trial in younger patients undergoing major non-cardiac surgery showed that the risk of postoperative complications was reduced if the systolic arterial pressure (SAP) was maintained at no more than $10 \%$ below the preoperative baseline, using individualised blood pressure management strategies. ${ }^{12}$ Other studies in various surgical populations showed an association between postoperative organ dysfunction and increasing duration and extent of intraoperative hypotension. ${ }^{13} 14$

At present there are no data correlating intraoperative hypotension with postoperative morbidity in patients undergoing hip fracture repair. In this observational study, we tested the hypothesis that duration and severity of intraoperative hypotension are predictors of postoperative morbidity in elderly patients undergoing hip fracture surgery.

\section{Methods}

\section{Chart review and quantification of baseline comorbidity}

We conducted a retrospective observational cohort analysis of all patients who underwent surgery for hip fracture repair in the Mater Misericordiae University Hospital, Dublin, Ireland between January and June 2017 inclusively. Patients were identified for inclusion in the study through the Hospital In-Patient Enquiry health information system, which generates a basic summary of administrative, clinical and demographic data whenever a patient is discharged from hospital or dies in hospital in Ireland. Patients were therefore included whether they presented through the emergency department from home, or were in-patients with other diagnoses who sustained hip fractures in hospital, as well as those transferred from nursing homes or long term care facilities. Some patients were transferred from other healthcare facilities without a trauma orthopaedic service. All patient clinical data were collected and stored on secured hospital computers with individual password protection for each investigator. Institutional review board approval was obtained for this retrospective analysis. 
Clinical records were retrieved for the identified patients, and were analysed for demographic and clinical data including age, gender, preoperative comorbidities, ASA status, length of stay in hospital,

comorbidities during the first 30 postoperative days, use of general or spinal anaesthesia, and use of noninvasive or invasive blood pressure monitoring. We described the cumulative preoperative comorbidity of our cohort using the Charlson Comorbidity Index; this internationally validated weighted scoring system stratifies patients according to expected in-hospital mortality based on their comorbid condition, assigning expected one-year mortality rates accounting for number and severity of comorbidities. ${ }^{13}$ Data were then correlated to intraoperative events stored electronically and accessed through Centricity High Acuity Anesthesia (GE Healthcare, Chicago, Illinois, United States), which is the anaesthesia information management system (AIMS) used in our institution to automatically record intraoperative events and measurements of patient vital signs throughout anaesthesia and recovery.

\section{Assessing duration of hypotension}

Blood pressure measurements were retrieved and analysed for each case from the time of induction of anaesthesia until the time the patient was transferred to the post-anaesthesia care unit. We applied several definitions for intraoperative hypotension:

1. cumulative time during which SAP was $\leq 80 \%$ of the initial measurement taken when the patient was clerked into hospital with a diagnosis of hip fracture;

2. cumulative time during which SAP was $\leq 80 \%$ of the last SAP measurement recorded prior to induction of anaesthesia;

3. cumulative time during which mean arterial pressure (MAP) was $<75 \mathrm{mmHg}$; and

4. cumulative time during which MAP was $<55 \mathrm{mmHg}$.

Where invasive arterial pressure monitoring was used, recordings had been logged by the AIMS every minute, and where non-invasive monitoring was used, recordings had been logged as per the clinical judgement of the anaesthesiologist caring for the patient, usually every three to five minutes. For each of our definitions for intraoperative hypotension, we defined duration of hypotension as the time between successive blood pressure measurements during anaesthesia where the patient's most recent recorded blood pressure met the respective criteria.

\section{Quantification of postoperative morbidity}

The patients' clinical records were analysed to ascertain the incidence of complications postoperatively during the first 30 postoperative days, or until discharge from hospital or death if this occurred sooner. We used a postoperative complications survey modified from that described by Bennett-Guerrero et al. ${ }^{14}$ Complications of all major organ systems were included: cardiac arrhythmia, myocardial infarction, sepsis, respiratory compromise, thromboembolic events, cerebrovascular events, new cognitive impairment, confusion, coma, renal impairment, liver dysfunction, need for transfusion, need for intensive care, wound dehiscence, pain limiting early post-operative mobilisation, and death. 
Postoperative morbidity data was expressed in terms of the Clavien-Dindo classification and Comprehensive Complication Index. The Clavien-Dindo classification qualitatively ranks the severity of a postoperative surgical complication according to the extent of corrective therapy required. ${ }^{15}$ We recorded the highest Clavien-Dindo classification associated with each patient based on the single most severe complication they encountered during the first 30 postoperative days. The Comprehensive Complication Index is based on the Clavien-Dindo classification, and allows a patient's overall morbidity to be quantified on a scale from 0 to 100 by integrating multiple weighted Clavien-Dindo classifications for different complications into a composite number. ${ }^{16}$

\section{Statistical analysis}

The dependent variables chosen for the prediction models were the Comprehensive Complication Index, which was treated as a continuous outcome variable, and the Clavien-Dindo index, which was treated as an ordinal outcome variable. Because of the limited number of cases per level, particularly at the higher end of the Clavien-Dindo index, the levels $3 a, 3 b, 4 a, 4 b$ and 5 were collapsed to a new level " $\geq 3$ ".

Univariate summary statistics were calculated for all independent and dependent variables.

The association between hypotension and post-operative complications were analysed by multivariable regression. As dependent variables the collapsed Clavien-Dindo classification and the Comprehensive Complication Index were chosen. The four previously mentioned cumulative time of hypotension variables of interest were included in analysis as continuous independent variables. Additional independent variables were also included due to their previously established association with postoperative complications:

- age at surgery; ${ }^{15,16}$

- gender; ${ }^{16,17}$

- preoperative morbidities as composed in the Charlson comorbidity index or the ASA status. ${ }^{16,18}$

To analyse the association between independent variables and post-operative complications a proportional odds models were chosen for the Clavien-Dindo index. The proportional odds assumption of the models was tested using a visual method described by Harrell and the likelihood ratio statistic. ${ }^{38,39}$ Linear models were chosen for the Comprehensive Complication Index. Relative parsimony between base and experimental models was determined using the corrected Akaike's Information Criterion $\left(\mathrm{AIC}_{\mathrm{C}}\right) .{ }^{19,20}$ Likelihood ratio tests were used to compare goodness-of-fit between models. $\mathrm{P}$ value of 0.05 or less was taken to indicate statistical significance for hypothesis testing. Statistical analyses were performed using the statistical software package R (R Development Core Team, Vienna, Austria). ${ }^{21}$

\section{Results}

Univariate and bivariate descriptive statistics 
$\mathrm{N}=65$ eligible patients were identified for inclusion in the study. Of these, $\mathrm{n}=13$ patients were excluded due to intraoperative data on the AIMS being unavailable or incomplete, due to their surgery not having taken place within the study period, or due to the patients not having proceeded to surgery. This left $n=52$ patients included in the full analysis. The characteristics of the patient cohort, together with duration of hypotension using our four definitions as well as lowest absolute values for SAP and MAP, as well as postoperative Clavien-Dindo classifications and Comprehensive Complication Index, are summarised in Table 1. $88 \%$ of patients had an ASA class of II or III, and $40 \%$ underwent general anaesthesia. Of those who received a spinal anaesthetic, $40 \%$ had invasive blood pressure monitoring. Comprehensive Complication Index ranged from 0 (no complications) to 100 (death), with a mean average score of 20.4 \pm 19 .2. Two patients had complications ranked as 4 on the Clavien-Dindo classification, meaning they suffered single or multiple organ failure subsequent to their operation, and one had a complication ranked as 5 (death).

Bivariate associations of the four cumulative time of hypotension variables against the Clavien-Dindo classification are shown in Figure 1. The clearest association is the increasing trend between cumulative time of SAP $<80 \%$ pre-induction baseline and the Clavien-Dindo classification.

Bivariate associations of the four cumulative time of hypotension variables against the Clavien-Dindo classification are shown in Figure 2. It is difficult to discern any trends between Comprehensive Complication Index and the cumulative time of hypotension variables, except for cumulative time of SAP $<80 \%$ pre-induction value, which showed a possible increasing trend.

\section{Multivariable analysis with the Clavien-Dindo index as outcome}

Multivariable regression analysis with the Clavien-Dindo index as dependent variable was performed. Four covariates for hypotension as listed in the Methods section were tested for their association with the Clavien-Dindo index, adjusted for base predictors (age, gender and Charlson Comorbidity score). The best fitting model included cumulative time of SAP $<80 \%$ pre-induction value as the covariate for hypotension ( $\mathrm{P}<0.001$ compared to other models). Adding time SAP $<80 \%$ pre-induction value to the model also significantly improved the goodness-of-fit over the base model that did not contain this covariate $(\mathrm{P}<$ 0.001), while also producing a more parsimonious model based on a lower $\mathrm{AlC}_{\mathrm{C}}$ over the base model.

As the Charlson Comorbidity Index and ASA classification have previously been shown to correlate with higher morbidity after hip fracture surgery, ${ }^{22}$ they were both tested as covariates for preoperative comorbidities by comparing these in separate models. The choice of covariate for preoperative comorbidity did not result in a significant improvement of goodness-of-fit of one base model over the other $(P=0.186)$. Due to the fewer degrees of freedom, the model with the Charlson Comorbidity index was more parsimonious.

The odds ratio of progressing to a higher Clavien-Dindo classification for a one-minute increase in cumulative time of SAP $<80 \%$ pre-induction value adjusted to gender, age and the Charlson Comorbidity 
Index was $1.020(95 \% \mathrm{Cl}: 1.008-1.035 ; \mathrm{P}=0.003)$. The coefficients of the model including their uncertainty are listed in Table 2.

\section{Multivariable analysis with the Comprehensive Complication Index as outcome}

Multivariable regression with the Comprehensive Complication index as dependent variable showed that also for this outcome, including cumulative time of SAP $<80 \%$ below pre-induction baseline as a covariate for cumulative time of hypotension produced the best fitting model, compared to models where the cumulative time of hypotension covariate was replaced with the other hypotension variables $(P<0.001$ compared to other models). Adding cumulative time of SAP $<80 \%$ pre-induction baseline to the model also significantly improved the goodness-of-fit over the base model $(P=0.002)$.

The null-hypothesis that goodness-of-fit of the models containing either the Charlson Comorbidity index or ASA as preoperative comorbidity index was not statistically significantly different was rejected, with the model with the former index showing a better fit $(P=0.048)$

For every additional minute of SAP $<80 \%$ pre-induction baseline, the mean Comprehensive Complication Index increased by 0.057 (95\% Cl: $-0.011-0.126 ; \mathrm{P}=0.102)$. However, this adjusted increase in mean was not statistically significant.

\section{Discussion}

A key challenge in caring for patients with hip fractures is expediting the corrective surgery while minimising the impact of preoperative morbidity and surgical stress, and thereby reducing the risk of postoperative complications. This objective has been delineated in guidelines from various bodies including the Scottish Intercollegiate Guidelines Network, the National Institute for Health and Clinical Excellence, and the Association of Anaesthetists. ${ }^{23-25}$ There is little evidence that generic prehabilitation or preoptimization strategies improve outcomes in hip fracture patients, but selected patients with serious intercurrent illness may benefit from a short period of medical management and stabilisation prior to their operation. ${ }^{26}$

Our data demonstrates a correlation between cumulative time of intraoperative hypotension, defined as a fall in SAP to less than $80 \%$ pre-induction baseline, and increased postoperative morbidity in this cohort of patients undergoing surgery for hip fracture. Despite a lack of consensus on how best to define hypotension, the Association of Anaesthetists has recommended using this definition as the "least bad" with respect to perioperative care of the elderly. ${ }^{23}$ No association between hypotension and postoperative morbidity was evident when the baseline for SAP was taken to be the first blood pressure measurement recorded for each patient on admission with their hip fracture. In our cohort, SAP on admission (most commonly in the emergency department) was significantly higher than the last SAP measurement taken prior to induction of anaesthesia; this likely reflects the fact that patients will have received analgesia and other medical therapies in the period between these two measurements, and blood pressure measurements taken prior to anaesthesia may therefore better reflect a patient's true baseline prior to 
their injury than blood pressure measurements in the emergency department. Evidence exists to support the assertion that blood pressure should be maintained as close to baseline as possible. One previous retrospective analysis of intraoperative data has suggested that transgression below a MAP threshold of $55 \mathrm{mmHg}$, even for short durations, is a sensitive predictor of postoperative acute kidney injury and myocardial injury. ${ }^{13}$ Another analysis has suggested that both relative and absolute reductions in MAP may be equally effective. ${ }^{24,27}$

Most studies on anaesthetic management of hip fracture patients have focused on whether anaesthetic technique (typically general versus spinal anaesthesia) affects postoperative mortality, with a clear signal that mortality after hip fracture surgery remains in the order of $6-8 \%$, regardless of anaesthetic technique. ${ }^{6,28}$ Recent evidence more commonly supports the use of spinal anaesthesia and regional techniques over general anaesthesia, as they are associated with reduced incidence of intraoperative hypotension. ${ }^{24}$ Observational studies have noted high incidence of intraoperative hypotension, despite the heterogeneity of definitions of hypotension used, which is directly associated with increased 30 day mortality. ${ }^{5}$ Combined spinal and general techniques may be associated with precipitous falls in blood pressure. ${ }^{11}$ Many studies have suggested an association between neuraxial anaesthesia for hip fracture patients and a reduction in instances of hypotension, but a systematic review with meta-analysis reported that neuraxial anaesthesia is not associated with reduction in 30-day mortality, although it is associated with reduced in-hospital mortality and reduced length of hospitalisation. Another cohort study found that hospitals with higher utilisation of neuraxial anaesthesia for hip fracture surgery had lower mortality rates after hip fracture surgery, but that the difference in survival was not attributable to the utilization of neuraxial anaesthesia itself; instead, this served as a marker for other hospital-level factors such as provider skill level, the degree of preoperative risk stratification, and avoiding preventable complications. ${ }^{29} \mathrm{~A}$ recent prospective randomised controlled trial in younger but higher risk patients found that patients randomised to receive maintenance of intraoperative arterial blood pressure close to baseline had significantly reduced postoperative major morbidity compared with patients randomised to receive standard intraoperative arterial blood pressure management. ${ }^{12}$

This cohort of frail patients who had sustained hip fractures were at a high risk of organ failure even before surgery, as illustrated by their high Charlson Comorbidity Index (mean 5.7, estimated 10-year survival $2-21 \%$ ). The present study suggests that the use of invasive blood pressure monitoring is associated with significantly less inadvertent hypotension than non-invasive pressure monitoring in such patients; the Association of Anaesthetists has previously recommended routine use of intra-arterial cannulation and transduction for this purpose in elderly patients, particularly for major or emergency surgery. ${ }^{23}$ One difficulty that arose during our analysis of the data was how to account for the intermittent nature of non-invasive monitoring of arterial pressure: if a patient had two hypotensive blood pressure readings three minutes apart, is it more helpful or accurate when quantifying hypotension to count this as a three minute period of continuous hypotension, or to record it as two separate instances of low bloodpressure readings? Our analysis was retrospective, and the use of invasive or non-invasive blood pressure monitoring was non-randomised and non-blinded in our cohort. Further prospective studies are warranted 
to demonstrate whether invasive monitoring is effective as a means of reducing the duration of hypotension.

Little data is available on what pharmacological agents, and what modes of administration, are most effective in treating hypotension during anaesthesia for hip fracture surgery. Many agents have been very well studied in the obstetric population. ${ }^{30}$ In this context, phenylephrine, metaraminol and noradrenaline infusions are all potential candidates for peripheral short term infusion strategies. ${ }^{31,32}$ Ephedrine can also be used by infusion and is effective, though it may lead to more episodes of dysrhythmia. ${ }^{33}$ Phenylephrine has been the agent of choice for treating spinal anaesthesia induced hypotension due to its primary vasoconstrictor action, while ephedrine preferentially acts to improve cardiac output. ${ }^{34}$ However, a recent prospective study on the effects of phenylephrine for anaesthesia induced hypotension showed that it was effective at increasing cardiac output in patients who were relatively hypovolemic. ${ }^{31}$ Many patients in the hip fracture cohort will have a degree of physiological beta-blockade secondary to age-related autonomic dysfunction, making alpha- 1 adrenoceptor agonists the treatment of choice. ${ }^{34,35}$ It is not clear whether hypotension is more reliably prevented in high-risk patients by administering intermittent boluses purely when deemed appropriate by the clinician, or by using a continuous infusion. Studies also focus on the importance of intravascular filling and the role of adequate preoperative loading with either colloid or crystalloid. ${ }^{36}$

Finally, the present study examined hypotension only during the intraoperative period, from the induction of anaesthesia until onward transfer of the patient's care in the post-anaesthesia care unit. The role of postoperative hypotension in relation to postoperative morbidity merits closer investigation. Guidelines have previously recommended that all patients with a preoperative estimated mortality in excess of $10 \%$ should be admitted to a level 2 or 3 critical care facility after their surgery, where continuous blood pressure monitoring (among other therapies) is available, although in many countries practical resource limitations will not always allow for this. ${ }^{37}$ Potential future technological developments in telemetry or wireless monitoring could offer novel solutions to this problem, and remote monitoring of at-risk patients is a prospective area of future investigation.

This study has some important limitations. The sample size of the study is small, particularly with respect to the number of parameters estimated. This significantly affects the estimation precision of the parameters of interest and makes it difficult to determine which cumulative time of hypotension variable is closer associated with postoperative comorbidities. Another limiting factor of the small sample size was the necessity to collapse ordinal variables in the study, limiting its generalisability, which is further affected by the use of a single centre for patient recruitment for the study. Another limitation is the dichotomisation of the blood pressure variables, which negatively affects the power of parameter estimation and limits the ability to include interactions between variables in the prediction models. Finally, the small sample size does not allow for validation of the prediction models on test cohorts, which increases the risk of overfitting and lowers the generalisability of the study's findings. For these reasons, the study results cannot be treated as more than exploratory. 


\section{Conclusions}

This exploratory, single-centre, retrospective analysis of elderly patients undergoing hip fracture repair demonstrated an association between the duration and extent of intraoperative hypotension and increased postoperative morbidity, irrespective of anaesthetic technique. The duration of hypotension while under anaesthesia is a good candidate for further study as a predictor of postoperative complications. Duration of time under anaesthesia where MAP $<55 \mathrm{mmHg}$ may be more predictive of postoperative complications than MAP $<75 \mathrm{mmHg}$. Defining hypotension as a reduction in SAP below $80 \%$ of the baseline immediately prior to induction of anaesthesia may be more predictive than either using the initial SAP on admission to hospital as the baseline, or using MAP $<55 \mathrm{mmHg}$ as the definition of hypotension.

The hypothesis that actively maintaining intraoperative blood pressure close to preoperative baseline values will reduce postoperative morbidity, compared with patients receiving standard intraoperative blood pressure management, warrants evaluation in a prospective, randomised controlled trial.

\section{Abbreviations}

SAP systolic arterial pressure

MAP mean arterial pressure

SD standard deviation

ASA American society for Anaesthesiologists

AIMS anaesthesia information management system

AlCc Akaike's information criterion

\section{Declarations}

Ethics approval and consent to participate

The Mater Misericordiae University Hospital institutional review board approved this retrospective analysis prior to commencement.

Consent for publication

Not Applicable.

Availability of data and material 
The data that support the findings of this study are available from Mater Misericordiae University Hospital but restrictions apply to the availability of these data, which were used under permission for the current study, and so are not publicly available. Data are however available from the authors upon reasonable request and with permission.

Competing interests

The authors declare that they have no competing interests.

\section{Funding}

There was no specific funding received for by any of the authors for this study.

\section{Authors' contributions}

RC collected patient data, collated and searched patients' file, interpreted and analysed results, drafted and edited the manuscript and tables, and is the corresponding author. GB undertook preliminary analysis of results and manuscript editing. GC collected data. SV interpreted results, undertook final statistical analysis and edited the manuscript. DB supervised and edited the manuscript. All authors have read and approve this manuscript.

\section{Acknowledgement}

The authors would like to acknowledge Dr Ni Mhuircheartaigh of Mater Misericordiae University Hospital for her help and support in data curation.

\section{References}

1. National Office of Clinical Audit. Irish Hip Fracture Database National Report 2016. Dublin November 2017.

2. Royal College of Physicians. National Hip Fracture Database annual report 2017. London 2017.

3. Agency for Healthcare Research and Quality. HCUPnet, Healthcare Cost and Utilization Project. https://hcupnet.ahrq.gov. Accessed 21 November 2018.

4. International Osteoporosis Foundation. Broken bones, broken lives: A roadmap to solve the fragility fracture crisis in Europe. October 2018.

5. Healthcare Quality Improvement Partnership. Falls and Fragility Fracture Audit Programme National Hip Fracture Database: Anaesthesia Sprint Audit of Practice London2014.

6. Patorno E, Neuman MD, Schneeweiss S, Mogun H, Bateman BT. Comparative safety of anesthetic type for hip fracture surgery in adults: retrospective cohort study. BMJ. 2014;348:g4022.

7. Boddaert J, Cohen-Bittan J, Khiami F, et al. Postoperative admission to a dedicated geriatric unit decreases mortality in elderly patients with hip fracture. PLoS One. 2014;9(1):e83795. 
8. Donegan DJ, Gay AN, Baldwin K, Morales EE, Esterhai JL, Jr., Mehta S. Use of medical comorbidities to predict complications after hip fracture surgery in the elderly. $J$ Bone Joint Surg Am. 2010;92(4):807-813.

9. Pincus D, Ravi B, Wasserstein D, et al. Association Between Wait Time and 30-Day Mortality in Adults Undergoing Hip Fracture Surgery. JAMA. 2017;318(20):1994-2003.

10. O'Donnell CM, McLoughlin L, Patterson CC, et al. Perioperative outcomes in the context of mode of anaesthesia for patients undergoing hip fracture surgery: systematic review and meta-analysis. $B r J$ Anaesth. 2018;120(1):37-50.

11. Wood RJ, White SM. Anaesthesia for 1131 patients undergoing proximal femoral fracture repair: a retrospective, observational study of effects on blood pressure, fluid administration and perioperative anaemia. Anaesthesia. 2011;66(11):1017-1022.

12. Futier E, Lefrant JY, Guinot PG, et al. Effect of Individualized vs Standard Blood Pressure Management Strategies on Postoperative Organ Dysfunction Among High-Risk Patients Undergoing Major Surgery: A Randomized Clinical Trial. JAMA. 2017;318(14):1346-1357.

13. Walsh M, Devereaux PJ, Garg AX, et al. Relationship between intraoperative mean arterial pressure and clinical outcomes after noncardiac surgery: toward an empirical definition of hypotension. Anesthesiology. 2013;119(3):507-515.

14. White SM, Moppett IK, Griffiths R, et al. Secondary analysis of outcomes after 11,085 hip fracture operations from the prospective UK Anaesthesia Sprint Audit of Practice (ASAP-2). Anaesthesia. 2016;71(5):506-514.

15. D'Apuzzo MR, Pao AW, Novicoff WM, Browne JA. Age as an independent risk factor for postoperative morbidity and mortality after total joint arthroplasty in patients 90 years of age or older. $J$ Arthroplasty. 2014;29(3):477-480.

16. Kim SD, Park SJ, Lee DH, Jee DL. Risk factors of morbidity and mortality following hip fracture surgery. Korean J Anesthesiol. 2013;64(6):505-510.

17. Al-Taki M, Sukkarieh HG, Hoballah JJ, et al. Effect of Gender on Postoperative Morbidity and Mortality Outcomes: A Retrospective Cohort Study. Am Surg. 2018;84(3):377-386.

18. Menzies IB, Mendelson DA, Kates SL, Friedman SM. The impact of comorbidity on perioperative outcomes of hip fractures in a geriatric fracture model. Geriatr Orthop Surg Rehabil. 2012;3(3):129134.

19. Burnham KP, Anderson DR. Model selection and multi-model inference : a practical informationtheoretic approach. 2nd ed. ed. New York ; London: Springer; 2002.

20. Hurvich CM, Tsai C-L. Bias of the corrected AIC criterion for underfitted regression and time series models. Biometrika. 1991;78(3):499-509.

21. R: A language and environment for statistical computing [computer program]. Vienna, Austria: $\mathrm{R}$ Foundation for Statistical Computing; 2004.

22. Smith T, Pelpola K, Ball M, Ong A, Myint PK. Pre-operative indicators for mortality following hip fracture surgery: a systematic review and meta-analysis. Age Ageing. 2014;43(4):464-471. 
23. Griffiths R, Beech F, Brown A, et al. Peri-operative care of the elderly 2014: Association of Anaesthetists of Great Britain and Ireland. Anaesthesia. 2014;69 Suppl 1:81-98.

24. National Clinical Guideline Centre. The Management of Hip Fracture in Adults. London, United Kingdom2011.

25. Scottish Intercollegiate Guidelines Network. Management of hip fracture in older people: A national clinical guideline. 2009.

26. Maxwell L, White S. Anaesthetic management of patients with hip fractures: an update. BJA Education. 2013;13(5):179-183.

27. Salmasi V, Maheshwari K, Yang D, et al. Relationship between Intraoperative Hypotension, Defined by Either Reduction from Baseline or Absolute Thresholds, and Acute Kidney and Myocardial Injury after Noncardiac Surgery: A Retrospective Cohort Analysis. Anesthesiology. 2017;126(1):47-65.

28. Sessler DI, Sigl JC, Kelley SD, et al. Hospital stay and mortality are increased in patients having a "triple low" of low blood pressure, low bispectral index, and low minimum alveolar concentration of volatile anesthesia. Anesthesiology. 2012;116(6):1195-1203.

29. Mclsaac DI, Wijeysundera DN, Huang A, Bryson GL, van Walraven C. Association of Hospital-level Neuraxial Anesthesia Use for Hip Fracture Surgery with Outcomes: A Population-based Cohort Study. Anesthesiology. 2018;128(3):480-491.

30. Mercier FJ, Auge M, Hoffmann C, Fischer C, Le Gouez A. Maternal hypotension during spinal anesthesia for caesarean delivery. Minerva Anestesiol. 2013;79(1):62-73.

31. Kalmar AF, Allaert S, Pletinckx P, et al. Phenylephrine increases cardiac output by raising cardiac preload in patients with anesthesia induced hypotension. J Clin Monit Comput. 2018;32(6):969-976.

32. Chen D, Qi X, Huang X, et al. Efficacy and Safety of Different Norepinephrine Regimens for Prevention of Spinal Hypotension in Cesarean Section: A Randomized Trial. Biomed Res Int. 2018;2018:2708175.

33. Moslemi F, Rasooli S. Comparison of Prophylactic Infusion of Phenylephrine with Ephedrine for Prevention of Hypotension in Elective Cesarean Section under Spinal Anesthesi: A Randomized Clinical Trial. Iran J Med Sci. 2015;40(1):19-26.

34. Lonjaret L, Lairez O, Minville V, Geeraerts T. Optimal perioperative management of arterial blood pressure. Integr Blood Press Control. 2014;7:49-59.

35. Critchley LA. Hypotension, subarachnoid block and the elderly patient. Anaesthesia. 1996;51(12):1139-1143.

36. Xie R, Wang L, Bao H. Crystalloid and colloid preload for maintaining cardiac output in elderly patients undergoing total hip replacement under spinal anesthesia. J Biomed Res. 2011;25(3):185190.

37. Royal College of Surgeons of England and the Department of Health. The higher risk general surgical patient. Towards improved care for a forgotten group. 2011. 
38. Harrell FE. Regression modeling strategies : with applications to linear models, logistic regression, and survival analysis. New York: Springer; 2001.

39. Christensen RHB. ordinal--Regression Models for Ordinal Data. R package version 2019.4-25. http://www.cran.r-project.org/package=ordinal/2019.

40. Watt J, Tricco AC, Talbot-Hamon C, et al. Identifying older adults at risk of harm following elective surgery: a systematic review and meta-analysis. BMC Med. 2018;16(1):2.

\section{Tables}

Table 1: Univariate patient characteristics and durations of hypotension for the study cohort. SA: spinal anaesthesia. GA: general anaesthesia. NIBP: non-invasive blood pressure. ORIF: open reduction with internal fixation. 


\begin{tabular}{|c|c|}
\hline Age, years & mean 77.9, standard deviation \pm 12.9 \\
\hline Female gender & $n=39,75 \%$ of total \\
\hline Length of stay in hospital, days & $n=28$, range $3-122$ \\
\hline ASA physical status classification & (\% of total) \\
\hline 1 & $n=4(8 \%)$ \\
\hline 2 & $n=20(38 \%)$ \\
\hline 3 & $n=24(46 \%)$ \\
\hline 4 & $n=1(2 \%)$ \\
\hline Data not available & $n=3(3 \%)$ \\
\hline Charlson Comorbidity Index & mean 5.712 , range $0-11$ \\
\hline Documented surgical procedure & (\% of total) \\
\hline Open reduction of hip with internal fixation & $n=8(15 \%)$ \\
\hline Hemiarthroplasty of hip & $n=23(44 \%)$ \\
\hline Dynamic hip screw & $n=8(15 \%)$ \\
\hline Gamma nail & $n=8(15 \%)$ \\
\hline Total arthroplasty of hip & $n=4(8 \%)$ \\
\hline Revision of total arthroplasty of hip & $n=1(2 \%)$ \\
\hline Anaesthetic technique & (\% of total) \\
\hline$S A$, NIBP monitoring & $n=19(37 \%)$ \\
\hline$S A$, arterial line & $n=12(23 \%)$ \\
\hline GA, NIBP monitoring & $n=4(8 \%)$ \\
\hline GA, arterial line & $n=17(33 \%)$ \\
\hline Data not available & $n=4(8 \%)$ \\
\hline Vasopressor boluses administered, number & (\% of total) \\
\hline 0 & $n=13(25 \%)$ \\
\hline 1 & $n=22(42 \%)$ \\
\hline 2 & $n=11(21 \%)$ \\
\hline 3 & $n=5(10 \%)$ \\
\hline 4 & $n=1(2 \%)$ \\
\hline Duration of hypotension, minutes & Mean (range) \\
\hline$M A P<55 \mathrm{mmHg}$ & $102.02(0-362)$ \\
\hline$M A P<75 \mathrm{mmHg}$ & $83.63(0-344)$ \\
\hline
\end{tabular}




\begin{tabular}{|l|l|} 
SAP $<80 \%$ baseline on hospital admission & $69.76(0-463)$ \\
SAP < 80\% baseline prior to induction of anaesthesia & $63.43(0-322)$ \\
\hline Lowest absolute blood pressure, $\mathrm{mmHg}$ & Mean (range) \\
SAP & $82.2(51-122)$ \\
MAP & $55.02(33-92)$ \\
\hline Highest single Clavien-Dindo classification & $11(21 \%)$ \\
0 & $11(21 \%)$ \\
1 & $20(38 \%)$ \\
2 & $7(13 \%)$ \\
3 & $2(4 \%)$ \\
4 & $1(2 \%)$ \\
\hline 5 & mean 20.4, standard deviation \pm 19.2, range 0-100 \\
\hline Comprehensive Complication Index & \\
\hline
\end{tabular}

Table 2. Proportional odds model with time SAP $<80 \%$ pre-induction baseline as a hypothesised predictor. ${ }^{*} \mathrm{P}$-value obtained from Wald's test statistic.

\begin{tabular}{|l|l|l|l|l|l|}
\hline \multicolumn{6}{|l|}{ Model } \\
\hline \multicolumn{7}{|l|}{ Time SAP <80\% pre-ind + Age at surgery + Gender + Pre-op Charlson Comorbidity Index } \\
\hline Coefficient & $\boldsymbol{\beta}$ & SE & Odds ratio & $\mathbf{9 5 \%} \mathbf{C l}$ & P-value* \\
\hline Intercept 0|1 & 3.314 & 2.078 & & & \\
\hline Intercept 1|2 & 4.562 & 2.099 & & & \\
\hline Intercept 2| $\geq 3$ & 6.939 & 2.225 & & & \\
\hline Time SAP <80\% pre-ind & 0.02017 & 0.00681 & 1.020 & $1.008-1.035$ & 0.003 \\
\hline Age at surgery & 0.06990 & 0.02591 & 1.072 & $1.023-1.134$ & 0.007 \\
\hline Pre-op Charlson Cl & -0.17153 & 0.10938 & 0.842 & $0.676-1.042$ & 0.117 \\
\hline Gender (Female) & -0.93429 & 0.74854 & 0.393 & $0.085-1.661$ & 0.212 \\
\hline
\end{tabular}

Table 3. Linear model with time SAP $<80 \%$ pre-induction baseline as a hypothesised predictor. *P-value obtained from Wald's test statistic. 


\begin{tabular}{|l|l|l|l|l|}
\hline \multicolumn{5}{|l|}{ Model } \\
\hline \multicolumn{5}{|l|}{ Time SAP <80\% pre-ind + Age at surgery + Gender + Pre-op Charlson Comorbidity Index } \\
\hline Coefficient & $\boldsymbol{\beta}$ & SE & $\mathbf{9 5 \%} \mathrm{Cl}$ & P-value \\
\hline Intercept & -21.754 & 17.266 & & \\
\hline Time MAP <55 mmHg & 0.057 & 0.034 & $-0.011-0.126$ & 0.102 \\
\hline Age at surgery & 0.582 & 0.200 & $0.181-0.984$ & 0.005 \\
\hline Pre-op Charlson Cl & -1.122 & 1.002 & $-3.140-0.895$ & 0.269 \\
\hline Gender (Female) & -2.518 & 6.249 & $-15.103-10.069$ & 0.689 \\
\hline
\end{tabular}

\section{Figures}
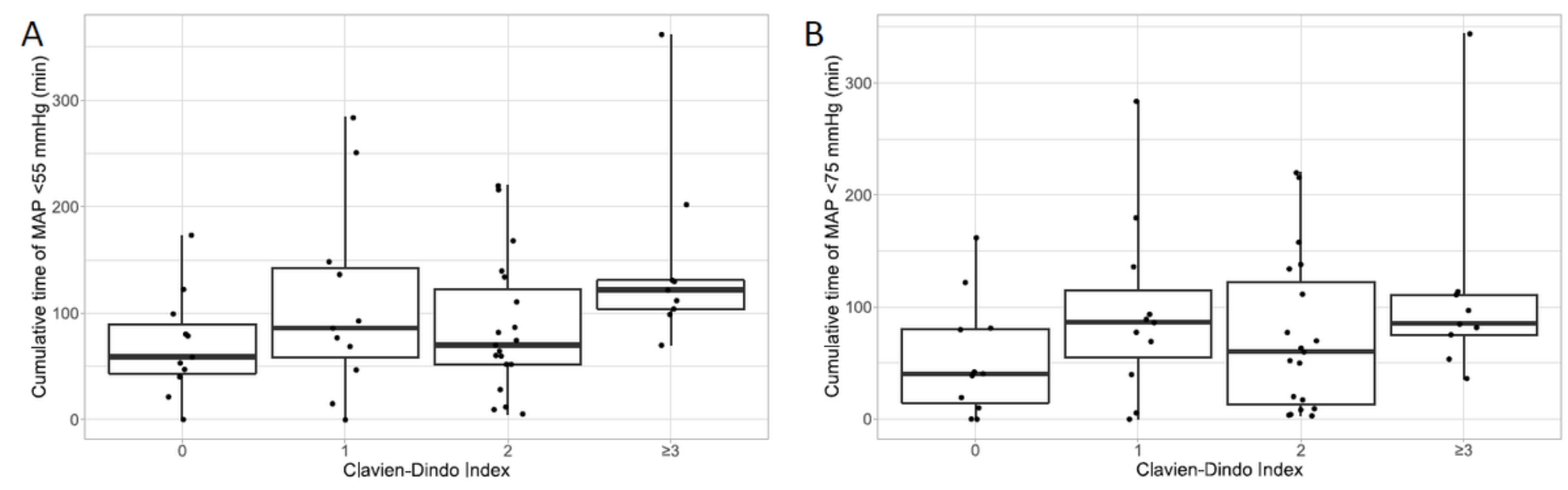

C

D
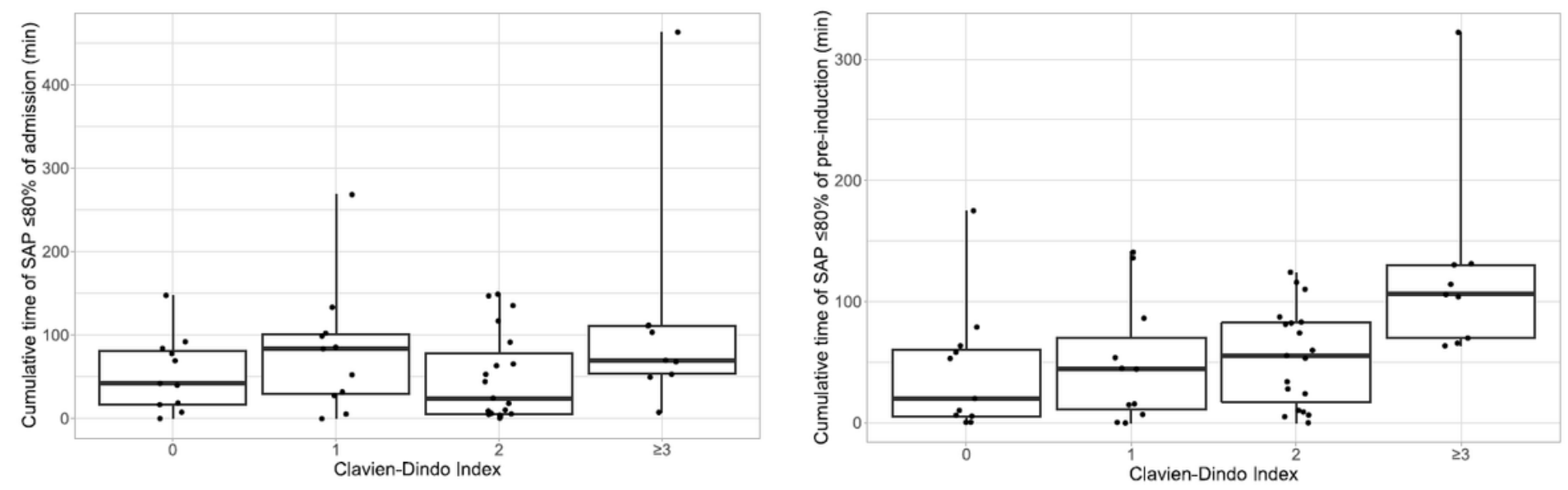

Figure 1

Descriptive bivariate analysis of hypotension variables against Clavien-Dindo index. (A) Time of MAP $<55$ mmHg, (B) Time of MAP $<75 \mathrm{mmHg}$, (C) Time of SAP $<80 \%$ admission baseline, (D) Time of SAP $<80 \%$ 
pre-induction baseline. In box plots the black horizontal bar represents the median, the box represents the interquartile range, the whiskers represent the range and the points the individual data.
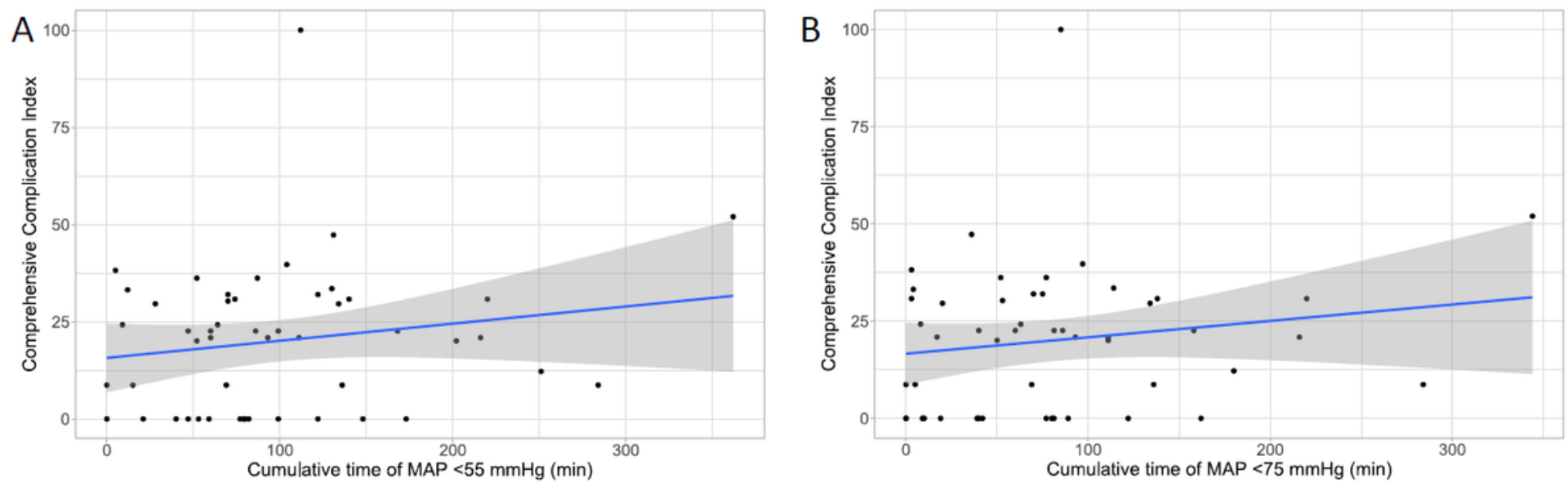

C

$\mathrm{D}$
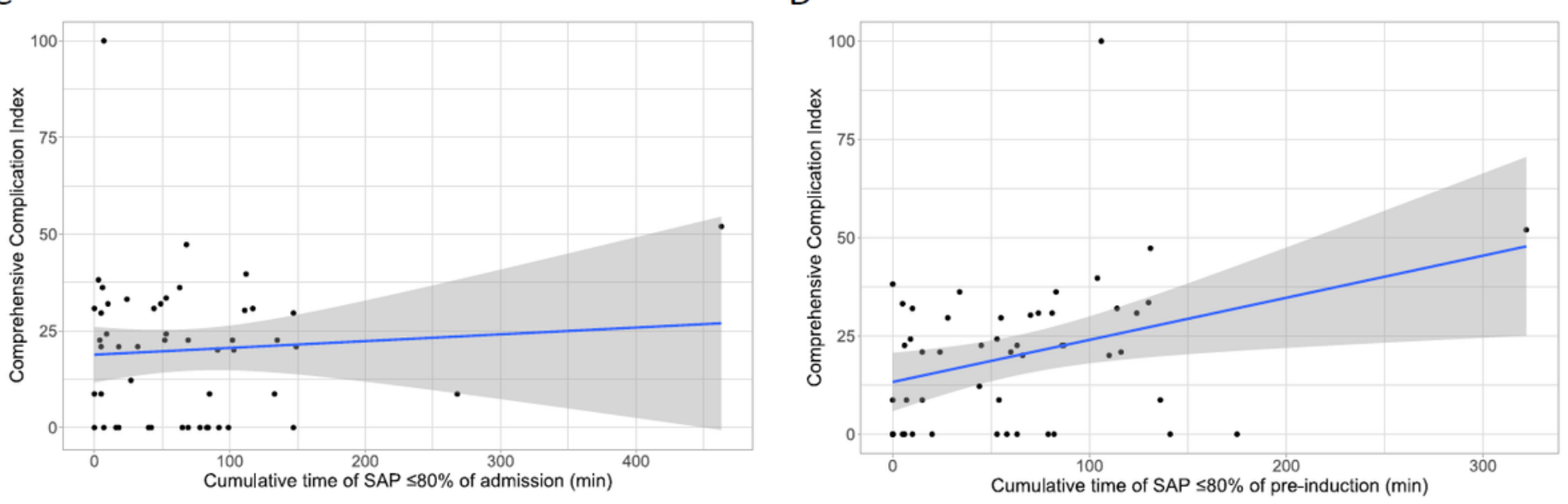

\section{Figure 2}

Descriptive bivariate analysis of hypotension variables against the Comprehensive Complication index at 30 days. (A) Time of MAP $<55 \mathrm{mmHg}$, (B) Time of MAP $<75 \mathrm{mmHg}$, (C) Time of SAP $<80 \%$ admission baseline, (D) Time of SAP $<80 \%$ pre-induction baseline. In the scatter plots, line represents the linear correlation as fitted by ordinary least-squares and the shaded area represents the standard error of this fit. 